\title{
Multi-layered Map based Navigation and Interaction for an Intelligent Wheelchair
}

\author{
Ruijiao Li, Lai Wei, Dongbing Gu, Huosheng Hu, Klaus D. McDonald-Maier
}

\begin{abstract}
Intelligent wheelchair is a paradigm of assisted living applications for elderly and disabled people. Its autonomous navigation and human-robot interaction is the major challenge. The previous intelligent wheelchair research has been mainly focused on geometric map based navigation, which is computational expensive in a large scale environment. This paper proposes the use of multi-layered maps for navigation and interaction of an intelligent wheelchair. The semantic information can improve the efficiency of path planning and navigation as well as extend the capability of task planning for the wheelchair. Some experimental results are given to demonstrate the feasibility and performance of the proposed approach.
\end{abstract}

\section{INTRODUCTION}

Our society is encountering a big healthcare problem from a global ageing population. Usually, elderly individuals suffer from mobility decline and other chronic diseases. They have difficulty with the daily life activities and must rely on healthcare and living assistance, which puts very heavy pressures to families and society [1]. The concept of Ambient Assisted Living(AAL) provides an excellent way to reduce the cost of healthcare and living assistance. AAL systems employ ICT-based technologies and deploy appliances and machines in living environment which can proactively and reactively accommodate users in their daily life.

Intelligent wheelchair is one of AAL applications that provides mobility service to people with motor impairment. Unlike the traditional wheelchairs, these intelligent wheelchairs are especially useful for those people with shoulder stroke, cognitive disease and apoplexy. During the last two decades, there are many research projects on intelligent wheelchairs. VAHM [2] is a long term EU project from 1989 to 2002 on intelligent wheelchair. An agent based wheelchair was developed during the VAHM project. NavChair [3] is an intelligent wheelchair research project founded at the University of Michigan in 1990. The collaborative control architecture was applied in NavChair and this significant architecture was deployed widely in many other intelligent wheelchairs [4].

The collaborative control method enables a wheelchair to work in a reactive way rather than only passively follows the command from the user. Many multiple modality humanmachine interfaces have been developed to adapt different user profiles. Wheelesley [5] is a wheelchair project at MIT

This work was supported by the EU Interreg IV A 2 Mers Seas Zeen Cross-border Cooperation Programme and The Colchester Catalyst Charity.

Authors are with the School of Computer Science and Electronic Engineering, University of Essex, Colchester CO4 3SQ, United Kingdom Emails: $\{r l i b$, lwei, dgu, hhu, kdm\}@essex.ac.uk
Artificial Intelligence Lab, which has deployed a multiple modal human-machine interface. Recently, a personal mobility and manipulation appliance (PerMMA) is proposed by Cooper [6]. It is a fully powered wheelchair on a mobile robot base with a custom track system around and two robotic arms. Its co-robot systems can accommodate everyday tasks such as fetch object, medicine service, open doors etc.

Previous research on wheelchair navigation has been mainly focused on geometric map based navigation. The complexity of geometric map based planning is computationally intensive. Nevertheless, it is difficult for people with cognitive impairment to operate such wheelchairs. On the other hand, deploying topological and semantic representation of an environment can significantly reduce the burden to human users. Recently, there has been an increasing interest in semantic mapping and cognitive robotics for assisted living.

In a semantic map, different places and objects are classified and categorized, such as common room, living room, kitchen, desk, bed, furniture, food etc. Each place and object has their own properties namely class, name, ID, function, pose, state. It also represents the topological relation of the components in the environment. Semantic spatial representation can enable a robot to interact with human through natural language and corresponding linguistics based label or text interface contains this information. A robot with semantic representation of heterogeneous places and objects can augment human-robot communication and interaction. Additionally, the semantic information can improve the efficiency of path planning and navigation as well as extend the capability of task planning for a robot.

Research in spatial representations for mobile robots shares a similar idea of multi-layered environment representation. Thrun [7] introduced a prototype of hybrid map which integrates metric map and topological map for in door robot navigation. Kuipers proposed [8] a paradigm Spatial Semantic Hierarchy (SSH) in 2000. It is regarded to be the precursor of multiple layered semantic spatial representation. Furthermore, Beeson et al. [9] introduced the Hybrid Spatial Semantic Hierarchy (HSSH) approach, which allows a mobile robot to describe the world using ontology and topological graph. Ontology representation is investigated for more than decades in information systems. It is the CoSy [10] project that employed ontology approach in robot system and developed a systematic and practical application in mobile robot system. Usually Semantic Web Ontology Language (OWL) is employed to store the representation of semantic map. 
This paper presents an interactive navigation system for an intelligent wheelchair with a multi-layered map prototype mentioned above. The geometric map provides the spatial information for localization. The topological map and geometric map are used for path planning. The semantic map representing the environment with an ontology model is used for human-robot interaction and task planning. Dialogue is used to provide a natural and friendly interface for humanwheelchair communication. A HTML5 based phone application is deployed for remote control of the wheelchair through a semantic map.

The rest of this paper is organized as follows. Section II introduces the mapping and navigation system for the wheelchair which is essential module of the wheelchair system. Section III is focused on the semantic representation of spatial information of the environment. Section VI presents some interaction approaches based on the multi-layered map and the autonomous navigation system. Some experimental results are given in Section $\mathrm{V}$ to show the feasibility and performance of our system. Finally, a brief conclusion and future work are given in Section VI.

\section{ROS BASED MAPPING AND NAVIGATION}

This section presents the hardware and the navigation system we developed. We aim to develop a universal wheelchair control system prototype that can be deployed to commercial electric-powered wheelchairs. Fig. 1 shows the electricpowered wheelchair with attached control components.

\section{A. Wheelchair System Overview}

The wheelchair system has two layered sections: low level section and high level section. The low level section consists of a Trim-slice PC with Nvidia Tegra 2 CPU and Linux system. The PC is connected with a joystick controller, a mode switcher, motors, and sensors (a Hukeyo laser range finder, a YEI-3D space sensor, two optical wheel encoders and an array of SONARs). A server programme running at the Trim-slice PC can publish sensor data and send commands to motors. The high level platform is a normal PC with Ubuntu Linux and ROS installed. It is connected with a Kinect (a RGB-D camera with audio input/output) and other HMI (Human-machine interface) devices. The two sections can communicate through TCP/IP server-client through WiFi connection. On the high level control platform, a client node acts as a message bridge and is wrapped into the ROS module as a comm_node which subscribes sensor data from low level server and publish the motion information generated by a navigation module.

\section{B. Geometric Map Building}

To reach a high level of autonomy, the robot needs the ability to build a map of the environment so that it can be used to locate and navigate safely. Geometric map contains metric spatial representation of an environment with frame reference and geometric relation. Our map building module is based on OpenSLAM gmapping in the ROS framework proposed by Gristti et. al. [11]. Gmapping provides effective laser based

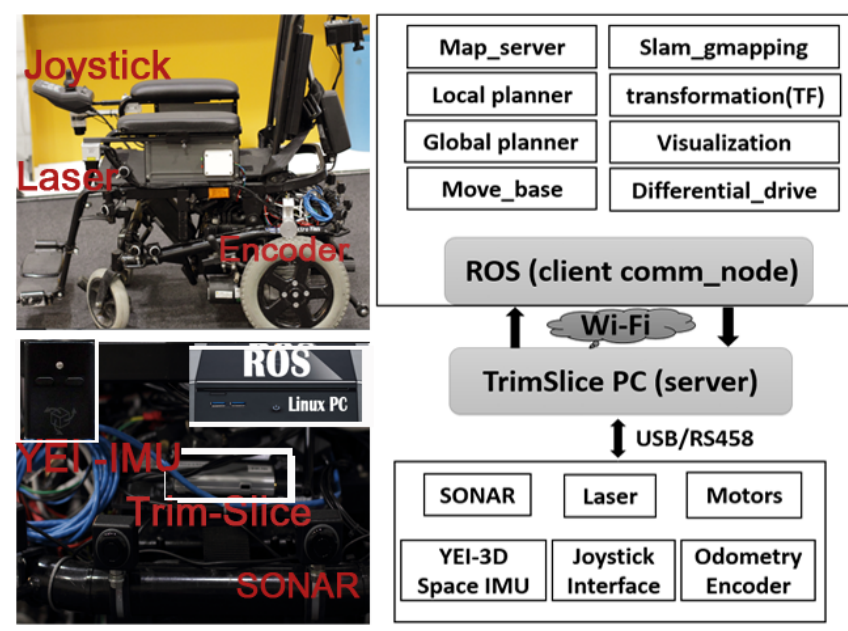

Fig. 1: Wheelchair Hardwares and ROS based control system

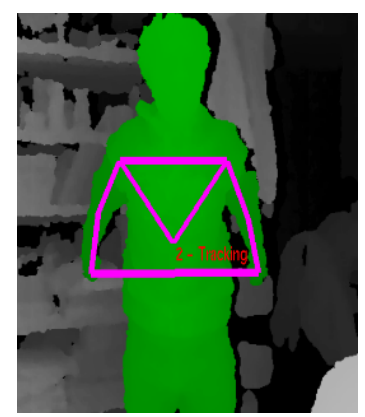

Fig. 2: Human body skeleton tracking with Kinect RGB-D sensor

Simultaneous Localization and Mapping (SLAM) method with the Rao-blackwellized particle filter approach and an adaptive particle reduction technique. Using Gmapping, we can create a $2 \mathrm{D}$ occupancy grid map from laser range data and odometry information of the wheelchair. Occupancy grid approaches disambiguate different places based on the robot geometric position within a global coordinate frame. The robot position is estimated incrementally, based on odometry information and sensor readings taken by the robot.

\section{Navigation}

The wheelchair navigation has two modes, namely person following mode and autonomous navigation mode. A 3D Kinect sensor is deployed on the wheelchair to track human motion. As shown in Fig. 2, human skeleton is detected by Kinect with OpenNI SDK. The range and orientation information from Kinect is used for the wheelchair to track and follow a person. The wheelchair motion speed and direction are determined by the kinect detection results. To prevent the risk of collision, the sonar sensors are used to detect the range to obstacles and stop the wheelchair once obstacle is detected in the range of threshold.

The scenario of autonomous navigation is, for a given goal pose, to produce safe path and send motion commands to the robot to reach the goal location by following the path 
according to the pre-built map and localization information. A probabilistic localization module, amcl, is used to track the pose of a robot against known maps. It implements the adaptive Monte Carlo localization approach with a particle filter to process the range and orientation information from sensors and maps.

For the wheelchair path planning, we employ mavfn package in ROS which wraps the $A^{*}$ heuristic algorithm for global path planning. A module, BaseLocalPlanner, within move_base is used for local planing. Map_server tool and YAML(a XML like format to specify map parameters) metadata decode the map data to a cost_map format which can be maintained by move_base for global planning and local planning. Once the motion commands for navigation is produced, the commands will be processed by a differential drive module with PID controller and publish them as motor commands to the low level platform. Besides the ROS navigation system, we have also developed a Bézier Curve Trajectory based doorway passage navigation module [12].

\section{Multi-Layered Spatial Representation}

Mozos [10], Zender [13] and Konolige [14] proposed the paradigm of a multi-layered map in their CoSy project. The multi-layered map aims to generate spatial and functional property of the environment, which enables a robot to perceive environments as human does. Such map enables robots to perceive the environment geometrically as well as exploit semantic representations, which can facilitate effective planning and human-robot interaction. This section describes how spatial representation of the environment is used for an intelligent wheelchair based on multi- layered map. As shown in Fig. 3, a multi-layered map consists of three layers: a geometric map at the bottom, a topological map in the middle to represent distributed areas in graph, and a semantic map at the top to represent properties and classes.

As mentioned above, grid-based occupancy maps can produce accurate spatial representation for robot localization and safe navigation. On the other hand, the complexity of grid map based planing is often very difficult in large scale environments. Thrun [15] introduced the metric-topological map approach for indoor mobile robot navigation which can be used much more efficiently, but also consistent to a gridbased map. Konolige et. al. [14] at Willow Garage designed an effective robot navigation system with a hybrid metrictopological map. Their system uses Dijkstras algorithm for planning on a pose-graph topological map and their result demonstrated that the hybrid map based planning is more effective than the metric based planning. Topological maps separate regions on top of a grid map. For instance, a building is classified into floors connected by lift, and each room on a floor is connected by corridor and door.

Mozos et al. [16] presented a method to extract a topological semantic map from a metric one using supervised learning. After the floor is classified into rooms, corridors, doors, then a pose graph [14] is used to connect regions to

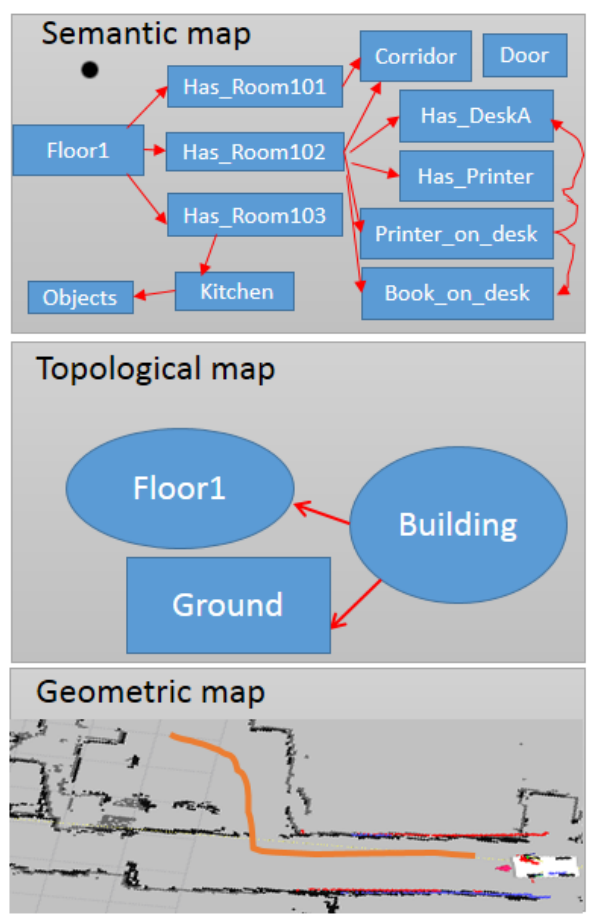

Fig. 3: Multi-layered hierarchy map

generate a topological map. The graph can be defined as $G=\{N, E, S, P\}$ where:

- $N$ is a set of nodes such as rooms, corridors, doors. Each node has a unique name i.e foor $\$ 1$, floor $\$ 2$, room $\$ 101$, room $\$ 102$, door $\$ 102$.

- $E$ is a set of edges which represents the constraint between nodes.

- $S$ refers to a set of current region of robot with regard to sensor data and odometry on a geometric map.

- $P$ is a set of global pose of each node.

With this topological map, the robot wheelchair can choose different navigation modules in different regions- For instance in a room, the navigation is controlled by a grid map based planner; once it reaches a door, the door passing module will take the control.

The top layer is semantic map, which includes a ontology based spatial concept of environments related to topological map and geometric map. It gives uniform meta-data description of environments as well as context of the objects within the environments. It provides a bridge between spatial information and human concept that enable situated and natural human-robot communication. Based on the work in [10] and Pronobis [17], the ontology knowledge base of an environment is combined with topology and geometry information. The ontology contains taxonomies and relationship of environments properties that allow the wheelchair to make inferences and reasoning about heterogeneous environments.

As shown in (Fig. 3, top), a building has floors and rooms. The rooms have different functions or types, e.g. common room, office, kitchen. i.e. room has_pose [pose $(\mathrm{x}, \mathrm{y}, \mathrm{z})]$, is_room \$101. room has_type commonRoom. Moreover, as 


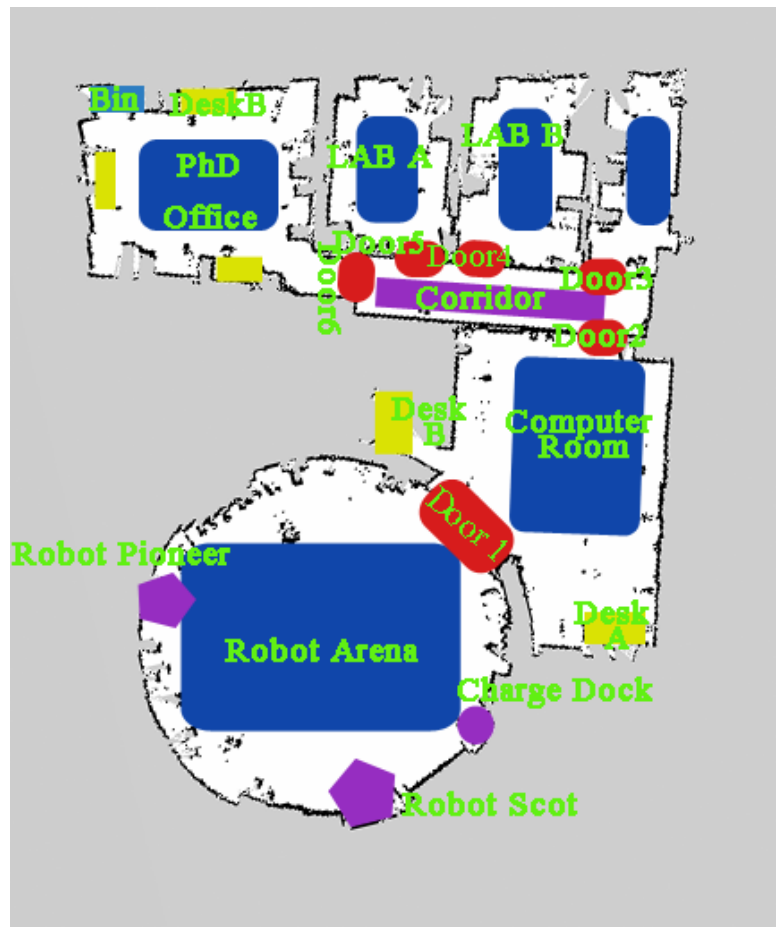

Fig. 4: Semantic map labeling paradigm

the paradigm indicated in Fig. 4, a room has some objects (printer, desk, PC), we say room has_object printer, has_object desk to describe the relation between room and object. For a object, it has properties has_id desk has pose $(\mathrm{x}, \mathrm{y}, \mathrm{z})$. The ontology description of an environment is implemented with OWL semantic formalisms.

With OWL description, for example, we can define the semantic representation of computer room as:

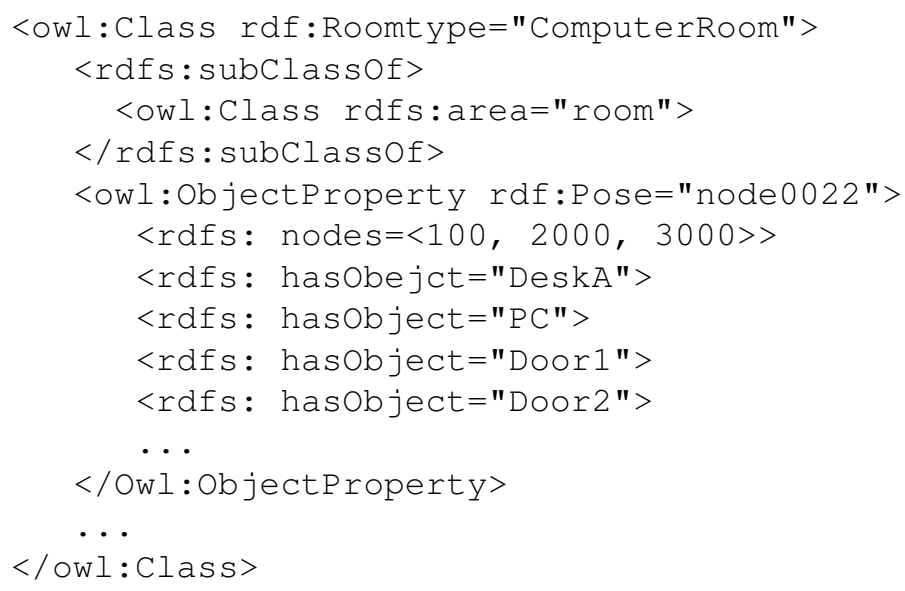

Basically, we define following concept to represent environment. Roomtype class is with regard to the function of rooms, such as computer room, Lab, common room, etc. Each object has class, properties such as pose, node, subclass; has Object is used to describe the spatial relationship of objects. The space is divided into three areas: room, corridor, door in class. Finally, when we have a semantic created by OWL, we can use the semantic map reasoning framework, knowrob, which includes Prolog interface to query OWL ontology knowledge base in ROS. Besides the taxonomies of the environment properties, we can also define task ontology with OWL i.e putting something on a desk will lead to a sequence of reasoning and actions.

\section{Multi-modal Human-machine Interaction}

In addition to a multi-layered map, we have proposed a sequence of interaction approaches with the wheelchair for assisted living. Fig. 5 presents the human-robot interaction module we have developed.

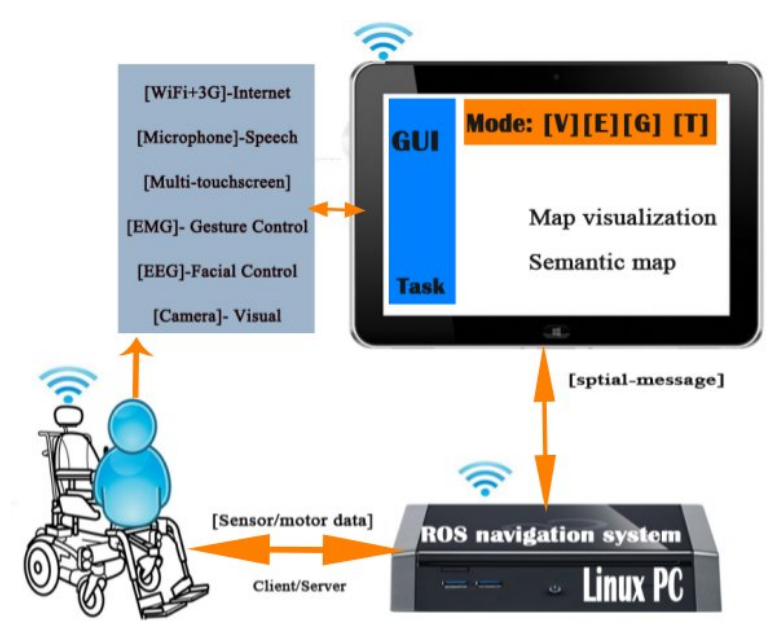

Fig. 5: Multi-modality interaction for the wheelchair

\section{A. Dialogue Interaction}

Following the system prototype proposed by [18] and [19], a dialogue system is designed for the wheelchair. CMU Sphinx speech recognition package [20] is employed for the dialogue interface. Some speech based command is defined for dialogue. Along with the speech commands, TTS (TextTo-Speech) package is used to provide voice instruction and feedback for the wheelchair.

- Pilot: Forward, Right, Left, Backward, Stop. It gives user freedom to drive the wheelchair with speech.

- Follow me command can trigger the person following module.

- Map update will start the interface to update the semantic map. Users can either type or say the name of objects or areas.

- Go to $\$ N A M E$ is defined to query the semantic map and to start the autonomous navigation module of the wheelchair to a specified location.

\section{B. Remote Interaction}

We also designed a remote control interface which enables a caregiver to help the wheelchair user remotely by his/her phone or tablet through the Internet. A HTML5 based phone application provides a user interface to send message to the ROS based wheelchair control system. A caregiver can touch the text panel or, choose speech mode and say the corresponding text to actuate a action on wheelchair i.e 
Go to $\$ N A M E$. Additional task planning based command will be endowed in future, for example, "take medicine" command can trigger the mounted arm on the wheelchair to take medicine for the wheelchair user.

\section{Hybrid Interfaces}

In order to make the wheelchair adapt to different user profile, we investigated different optional interaction approaches. Facial emotion control, head gesture control, EMG based hand motion control have been developed and tested on our intelligent wheelchair. Moreover, the control commands produced by these control method can be mapped to the element of a semantic map to generate relevant action predefined.

\section{EXPERIMENTS}

The preliminary test has been carried out on our wheelchair and ActiveMobile Pioneer3DX. Since the odometry of the wheelchair is not accurate, we deployed the same system on a Pioneer robot. For wheelchair and Pioneer robot, we just need to configure the transformation and communication interface within ROS.

\section{A. SLAM mapping}

Fig. 6 presents the maps built by SLAM gmapping of the wheelchair in our research Essex Robotics Research LAB. Fig. 7 shows the map built by the Pioneer robot. The environment was mapped by joystick controlled exploration and laser scanning through the LAB. Then the logged data is processed by SLAM gmapping and produce map by ROS map server. Comparing the two maps by generated by the wheelchair and the Pioneer robot with same control system, the map is lack of accuracy since the odometry error. We are developing a IMU-based odometry and heading reference module to correct the wheel encoder for odometry.

\section{B. Supervised Semantic Mapping}

The semantic map is generated upon the grid map through makers and supervised labeling. Fig. 8 shows the map and markers. When we click a marker, its pose $(x, y, z)$ with corresponding ID is stored in a logged file. The area is classified as room, corridor, and door. The ontology knowledge base can be updated with the properties of the logged data.

\section{Interactive Navigation}

Fig. 9 shows some experimental results with a real robot in Essex Robotics Research Laboratory which consists of a circular lab space, a near rectangle lab space, 4 offices and 1 long corridor. To test the interactive navigation, a goal message is sent to to the navigation module of the robot. Then the path planing module generates a collision free route for navigation. The yellow cells indicate the obstacles and the orange color cells represent the obstacles in the visualized map. The blue curve in the figures represent the trajectories of the robot, in (a), doorl is a goal from the dock sent by message. In (c), the robot moves from the circular lab to an office via the corridor. In Fig. 9(d), the blue line indicates the navigation trajectory for the robot to return the dock point.

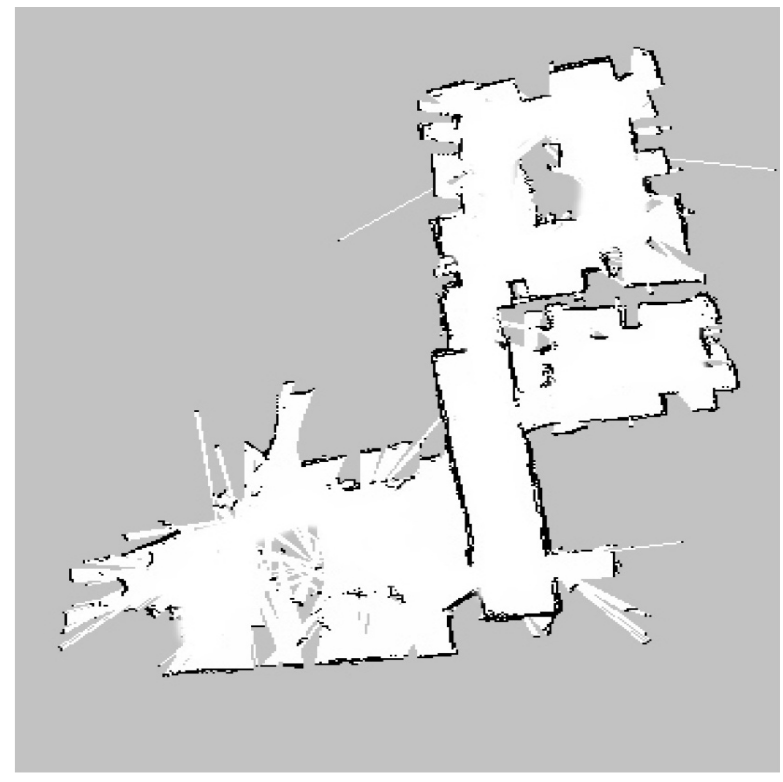

Fig. 6: Map built by a wheelchair

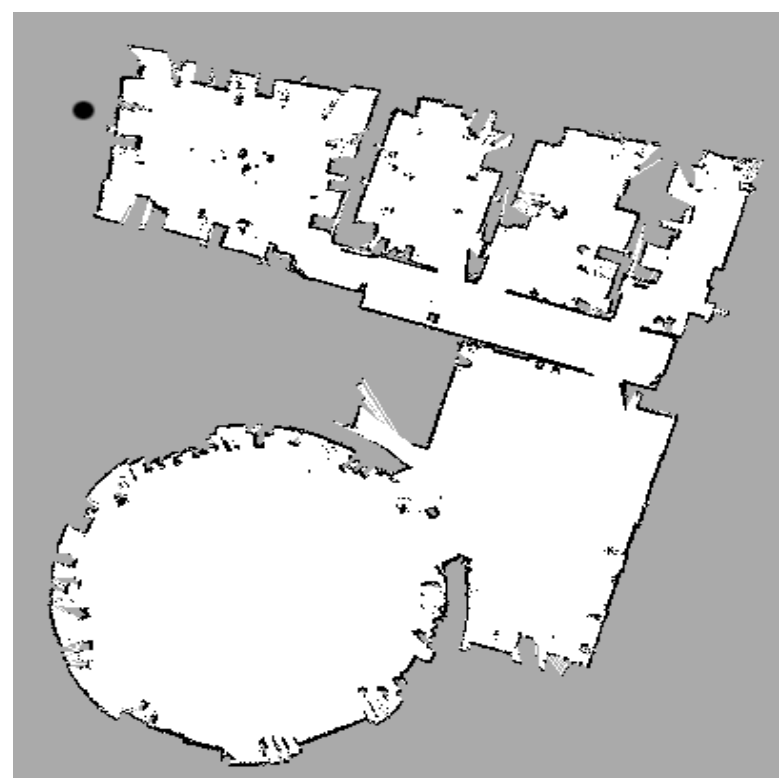

Fig. 7: Map built by a Pioneer robot

\section{DISCUSSION AND CONCLUSIONS}

In this paper, we have addressed how a multi-layered map could be used for intelligent wheelchair navigation and how its semantic contents could be used for humanwheelchair interaction. A commercial wheelchair has been equipped with multiple sensors and embedded computers for this research. A ROS-based autonomous navigation system is deployed for the control and navigation of the wheelchair. A multiple layered map has been built for effective interaction and navigation by integrating a geometric map, a topological map and a semantic ontology map. Human-robot interaction is realized at the top of the system.

The preliminary implementation of the proposed system has demonstrated the feasibility and performance of mapping 


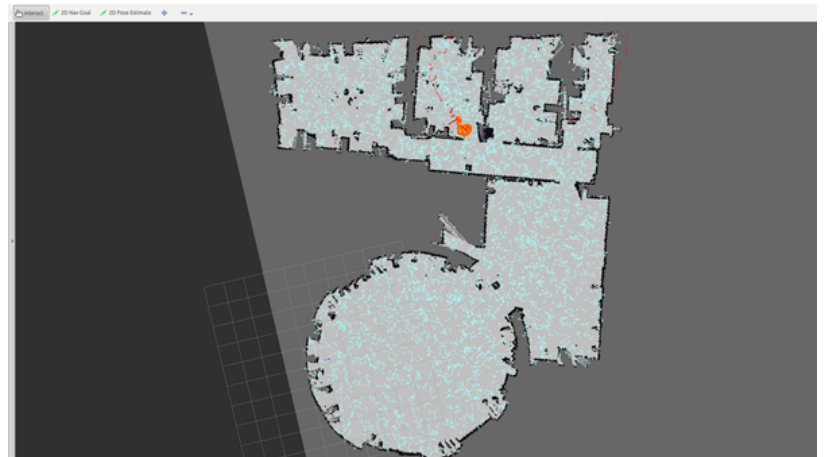

Fig. 8: Map visualization and maker point cloud in ROS RViz

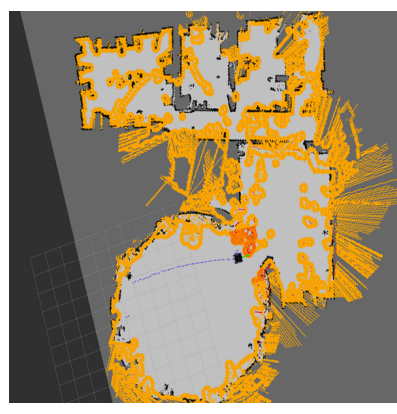

(a)

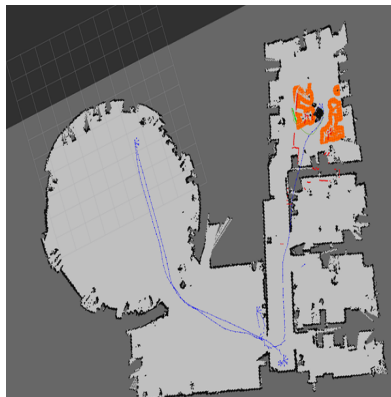

(c)

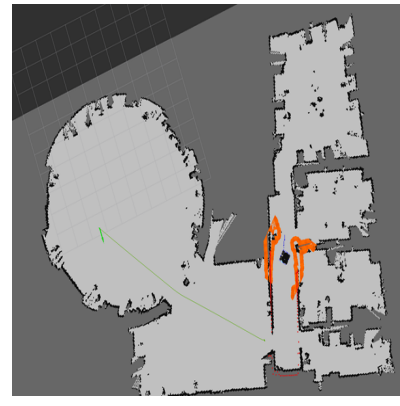

(b)

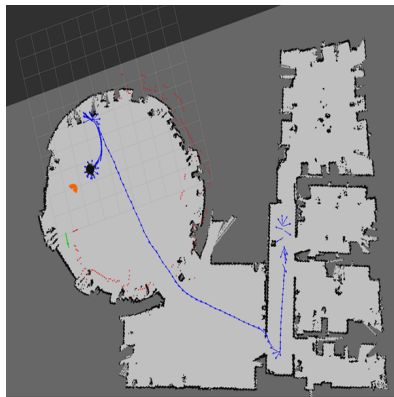

(d)
Fig. 9: Autonomous navigation and trajectory visualization

and interactive navigation of such a semantic world model. However, the current system is not accurate and robust since its sensor limitation. In the future, a 9-axis IMU will be deployed with odometry to improve the navigation accuracy. Also, a bridge will be developed to send speech commands to the ROS framework. A further scientific question is how to realize automatic 3D environment classification and semantic representation for assisted living by developing an advanced and robust cognitive system.

\section{ACKNOWLEDGMENTS}

This research is financially supported by the EU Interreg IVA 2 Seas Programme (SYSIASS project: Autonomous \& Intelligent Healthcare System, http://www.sysiass.eu/), and Franco-British programme (COALAS Project http://coalasproject.eu/) that has been selected in the context of the
INTERREG IVA France (Channel) England European crossborder co-operation programme, which is co-financed by the ERDF. Our thanks also go to Colchester Catalyst Charity for their financial support, Robin Dowling and Ian Dukes for their technical support.

\section{REFERENCES}

[1] UN, "World population ageing: 1950-2050." [Online]. Available: http://www.un.org/esa/population/publications/worldageing19502050/

[2] G. Bourhis and P. Pino, "Mobile robotics and mobility assistance for people with motor impairments: rational justification for the VAHM Project," IEEE transactions on Rehabilitation Engineering, vol. 4, no. 1, pp. 7-12, Mar. 1996.

[3] S. Levine, D. Bell, and Y. Koren, "NavChair: An example of a shared-control system for assistive technologies," in Computers for Handicapped Persons, 1994.

[4] T. Carlson and Y. Demiris, "Collaborative control for a robotic wheelchair: evaluation of performance, attention, and workload." IEEE transactions on systems, man, and cybernetics. Part B, Cybernetics : a publication of the IEEE Systems, Man, and Cybernetics Society, vol. 42, no. 3, pp. 876-88, Jun. 2012.

[5] H. Yanco, "Wheelesley: A robotic wheelchair system: Indoor navigation and user interface," Assistive technology and artificial intelligence, 1998.

[6] R. Cooper, G. Grindle, and J. Vazquez, "Personal Mobility and Manipulation ApplianceDesign, Development, and Initial Testing," Proceedings of the IEEE, 2012.

[7] S. Thrun and A. Bücken, "Integrating grid-based and topological maps for mobile robot navigation," in Proceedings of the National Conference on Artificial Intelligence, no. 8, 1996.

[8] B. Kuipers, "The spatial semantic hierarchy," International Journal of Artificial Intelligence, vol. 119, pp. 191-233, 2000.

[9] P. Beeson, J. Modayil, and B. Kuipers, "Factoring the Mapping Problem: Mobile Robot Map-building in the Hybrid Spatial Semantic Hierarchy," The International Journal of Robotics Research, vol. 29, no. 4, pp. 428-459, May 2009.

[10] O. M. Mozos, A. Rottmann, and R. Triebel, "Semantic labeling of places using information extracted from laser and vision sensor data," in IEEE/RSJ IROS 2006 Workshop: From Sensors to Human Spatial Concepts (IROS), 2006.

[11] G. Grisetti, C. Stachniss, and W. Burgard, "Improved Techniques for Grid Mapping With Rao-Blackwellized Particle Filters," IEEE Transactions on Robotics, vol. 23, no. 1, pp. 34-46, Feb. 2007.

[12] S. Wang, L. Chen, H. Hu, and K. McDonald-Maier, "Doorway Passing of an Intelligent Wheelchair by Dynamically Generating Bézier Curve Trajectory," in IEEE International Conference on Robotics and Biomimetics, 2012, pp. 1206-1211.

[13] H. Zender, P. Jensfelt, and O. Mozos, "An integrated robotic system for spatial understanding and situated interaction in indoor environments," in Proceedings of the 22nd national conference on Artificial intelligence, 2007, pp. 1584-1589.

[14] K. Konolige, E. Marder-Eppstein, and B. Marthi, "Navigation in hybrid metric-topological maps," in 2011 IEEE International Conference on Robotics and Automation. IEEE, May 2011, pp. 3041-3047.

[15] S. Thrun, "Learning metric-topological maps for indoor mobile robot navigation," Artificial Intelligence, vol. 99, pp. 21-71, 1998.

[16] O. Mozos and W. Burgard, "Supervised Learning of Topological Maps using Semantic Information Extracted from Range Data," 2006 IEEE/RSJ International Conference on Intelligent Robots and Systems, pp. 2772-2777, Oct. 2006.

[17] A. Pronobis and P. Jensfelt, "Understanding the real world: Combining objects, appearance, geometry and topology for semantic mapping," Royal Institute of Technology (KTH) SE-100, Tech. Rep., 2011.

[18] E. A. Topp and H. Helge, "HRI Pilot study: Explicit environmental representations in the context of Human Augmented Mapping," in Proceedings of the 1st ACM Human Robot Interaction Conference, Salt Lake City, 2006, pp. 1-3.

[19] M. Walter, S. Hemachandra, and B. Homberg, "Learning Semantic Maps from Natural Language Descriptions," in Proceedings of Robotics: Science and Systems (RSS), 2013.

[20] CMU, "Sphinx speech recognition toolkit," Accessed on Aug. 5, 2013 . [Online]. Available: http://cmusphinx.sourceforge.net/ 